\title{
The enhancement of submandibular gland branch formation on chitosan membranes
}

\author{
Tsung-Lin Yang ${ }^{\mathrm{a}, \mathrm{b}}$, Tai-Horng Young ${ }^{\mathrm{a}, *}$ \\ ${ }^{a}$ Institute of Biomedical Engineering, College of Medicine and College of Engineering, National Taiwan University, Taipei, Taiwan \\ ${ }^{\mathrm{b}}$ Department of Otolaryngology, National Taiwan University Hospital and College of Medicine, Taipei, Taiwan
}

Received 12 November 2007; accepted 13 February 2008

Available online 3 March 2008

\begin{abstract}
Many glandular organs are developed by branching morphogenesis, an efficient and ubiquitous process for creating a larger cellular area for metabolic requirement. To regenerate a glandular organ, such as salivary glands, recapitulation of branching processes may be requisite. At present, the roles of biomaterials in regenerative branching have never been thoroughly explored. By culturing the embryonal submandibular gland (SMG) on different substrata, including polyvinyl alcohol (PVA), chitosan, and polycarbonate (PC), this study demonstrated for the first time that chitosan was capable of providing a more preferential environment for salivary gland branch formation. After culturing SMG explants on chitosan membranes, secreted extracellular matrices distributed in a reticular manner and formed thicker fibers beyond the extents of cell attachment, which were not found in PVA and PC. In the subsequent culture of explants just on the used chitosan substrates, these conditioned membranes were able to further enhance SMG branching. The fact that the promoting effects were eliminated with collagenase treatment and type I and type III collagen were identified within the adherent fibrillar extracellular matrix raised the possibility that the stimulating factors were collagen-originated. This indicates that, for SMG, chitosan is a bioactive substratum which enables cells to synthesize and deposit essential extracellular matrix, paving an important way for ensuing branch formation. Accordingly, the current study provides a larger scope to use chitosan as a biomaterial for recapitulating branching, which might be useful for the scaffold design of salivary gland regeneration.
\end{abstract}

(C) 2008 Elsevier Ltd. All rights reserved.

Keywords: Salivary glands; Submandibular gland (SMG); Biomaterials; Chitosan; Collagen; Branching morphogenesis

\section{Introduction}

Branching morphogenesis is a ubiquitous developmental process utilized by different tissues to form complex ramified structures. Although different in histological structures and physiological functions, they share similar mechanisms of branching with some tissue-specific features [1]. The glandular tissues benefit from branching processes by creating a larger cellular area for metabolic requirement within a limited space. For example, the submandibular gland (SMG), a pivotal organ in saliva secretion and regulation, is formed by branching morphogenesis. During development, SMG is formed from

\footnotetext{
* Corresponding author. Tel.: +886 2 23123456x1455; fax: +886 2 23940049.

E-mail address: thyoung@ntu.edu.tw (T.-H. Young).
}

a condensation of oral epithelium by embryonic day 12 [2]. The highly branched SMG is completed after repetitive processes of cleft invagination and buds sprouting in the ensuing developmental stages [3]. Finally, the bush-like structure creates enough cellular metabolic surface for SMG and makes it competent to meet the physiological demand [2-4].

Clinically, salivary dysfunction is a common issue and causes a significant morbidity, especially in the patients with irreversible salivary gland change from diseases or therapeutic managements. The destruction of salivary gland leads to xerostomia, a condition which annually happens in 500,000 people worldwide [5]. The hypofunctional salivary gland might impair oral hygiene, digestion, dental health, mucosal regeneration, and nutrition [6]. They altogether largely deteriorate the quality of life. Nonetheless, to date, most treatments aim at saliva substitution instead of the repair of dysfunctional salivary 
glands [7]. In treating a dysfunctional salivary gland, replacement with new functional tissues may be more physiological. For this purpose, many artificial and natural substrata have been examined for the biomaterial approach of salivary glands' regeneration [8,9]. Although with progress in the culture of salivary gland cells supported by scaffold, few reports have focused on the branching morphogenesis, another important issue of functional salivary glands. To recover functional salivary glands, the achievement of enough numbers of functional units and the controlled formation of glandular structures are requisite. Without successful branching, the amount of cell number and space for reconstituting a functional salivary gland increases to a greater extent.

Although much effort has been made to clarify the underlying mechanisms accounting for salivary gland branching morphogenesis, most studies have mainly addressed the growth factors and intrinsic signaling pathway instead of the cultured scaffold for organ regenerative purposes [2,4]. Previously, polycarbonate (PC) has been routinely used as the culture materials for investigating SMG branching $[2,4]$. However, the ex vivo cultured SMG sprouts fewer branches compared to that of in vivo development by the same stage, despite the addition of well-known branching-promoting factors [2]. Moreover, for organ regeneration, non-biodegradable PC is not regarded as an appropriate biomaterial. Thus, to recapitulate branching processes, the effects of cultured scaffold should be prudently evaluated.

Chitosan is a linear polysaccharide with a variable number of randomly located $N$-acetyl-glucosamine groups in structure, and characteristics of biodegradable, biocompatible, bioadhesive, non-toxic, non-antigenic, and tailorable properties [1012]. These desirable features render chitosan more accessible as matrices for tissue engineering applications [13,14]. Though chitosan has many advantages, its utility in regenerative salivary glands has seldom been addressed. In a previous study, salivary gland cells have been shown to grow well and preserve original phenotypes on the chitosan membranes [15]. The results suggest that chitosan might be a potential applicable scaffold in salivary gland regeneration.

The present study was undertaken to provide insight into the effects of chitosan on salivary gland branching that could be applied in the approaching strategies for the regeneration of glandular tissues. This study demonstrated for the first time that chitosan was capable of providing a more preferential environment for salivary gland branching morphogenesis. The current work is encouraging since this culture system may set the stage for future investigations of salivary glands' regeneration and offer a future option for patients with salivary dysfunction.

\section{Materials and methods}

\subsection{Preparation and characterization of membranes}

The substrates used in this study were prepared in the membranous forms as follows. For preparing PVA membrane, an appropriate amount of PVA (BF-17, Chang-Chun, Taipei, Taiwan) was dissolved in water to form a polymer solution. The polymer concentration for PVA was $14 \mathrm{wt}$.\%. In addition, a $2 \mathrm{wt}$ \% solution of chitosan was prepared by dissolving chitosan (448869, MW: $612 \mathrm{kDa}$, degree of deacetylation: $75-85 \%$, Sigma-Aldrich, St. Louis, MO, USA) in $1 \mathrm{M}$ acetic acid. Subsequently, the polymer solution was spread on a glass plate in the thickness of $500 \mu \mathrm{m}$ and was evaporated in a convection oven at $50{ }^{\circ} \mathrm{C}$ over $24 \mathrm{~h}$. During this time of evaporation, the casting solution became a solid membrane. Finally, the residual solvent in the nascent membranes was removed by a series of washing steps. In addition, commercial PC membranes (Crossbond, Taipei, Taiwan) were used for comparison. Before being used in explants' culture, these membranes were sterilized in $70 \%$ alcohol overnight under ultraviolet light and rinsed extensively with phosphate buffered saline (PBS).

\subsection{Ex vivo organ culture of SMG explants}

SMG explants were dissected from mid E12 ICR mice. Animal protocols were approved by the National Taiwan University Animal Care and Use Committee. To culture SMG explants on the indicated membranous substrata, the culture system was prepared as previously described with some modifications [4]. The explants were placed directly on the membranous substrata. The medium was added and carefully adjusted for amount to keep the explants submerged in the culture medium with an air/medium interface [16]. A silicone O-ring was placed onto the indicated membrane in each well to prevent the membrane from floating. The culture medium used for SMG explants was composed of DMEM/F12 medium supplemented with $100 \mathrm{U} / \mathrm{ml}$ penicillin, $100 \mu \mathrm{g} / \mathrm{ml}$ streptomycin, $150 \mu \mathrm{g} / \mathrm{ml}$ vitamin $\mathrm{C}$, and $50 \mu \mathrm{g} / \mathrm{ml}$ transferrin as described [4]. The medium was replenished every $8 \mathrm{~h}$. The SMG explants were cultured at $37{ }^{\circ} \mathrm{C}$ in a humidified $5 \% \mathrm{CO}_{2}$ and $95 \%$ air atmosphere. The morphologies of SMG explants were photographed and counted for branches at the indicated time points thereafter. The numbers of branches were averaged after at least three independent experiments.

\subsection{Scanning electron microscopy}

To characterize the used membranes, the conditioned PVA, chitosan, and PC membranes were examined using a Hitachi S-2600H scanning electron microscope (SEM). The conditioned membranes were washed with PBS after culture and then fixed with $2.5 \%$ glutaraldehyde in PBS at $4{ }^{\circ} \mathrm{C}$. After being thoroughly washed with PBS, the membranes were dehydrated by graded ethanol changes and dried. The specimens were then sputtered with gold in vacuum and photographed under SEM.

\subsection{Preparation and culture of SMG explants on conditioned membranes}

SMG explants dissected from mid E12 ICR mice were cultured on the conditioned membranes in the similar manner mentioned above. After culture, the explants were removed by gently washing with medium. The sites of membrane where the explants were placed were marked as the conditioned area. Subsequently, another freshly dissected SMG explant was placed exactly on the marked site of conditioned membrane for ex vivo culture. The other paired explant for control was placed at another site on the similar substrata without being cultured with explants. In the assay of conditioned membrane treated with collagenase, the conditioned membranes were treated with collagenase (C2674, Sigma-Aldrich, St. Louis, MO, USA) of $0.5 \mathrm{mg} / \mathrm{ml}$ concentration for half an hour. After incubation, the membranes were washed with PBS thoroughly to remove the residual collagenase. Subsequently, another freshly dissected SMG explant was just placed at the marked site of conditioned membranes. Another paired one for control was cultured in the same manner All explants on conditioned and control membranes were cultured at $37{ }^{\circ} \mathrm{C}$ in a humidified $5 \% \mathrm{CO}_{2}$ and $95 \%$ air atmosphere. The morphologies of SMG explants were photographed at the indicated time points and counted for average after at least three independent experiments.

\subsection{Immunohistochemistry and quantification of collagen deposition}

The deposition of protein on the indicated membranes was detected by the method of enzyme-linked immunosorbent assay (ELISA) [17]. Membrane substrates which have been used for SMG culture were cut and fixed with 
4\% paraformaldehyde in PBS and washed. Then each substratum was rinsed with PBS. To detect protein deposition on indicated membrane, the associated culture substrata were pretreated with $10 \%$ bovine serum albumin in PBS for $2 \mathrm{~h}$ at $37^{\circ} \mathrm{C}$. After the surface was washed and rinsed with blocking buffer, the corresponding antibody was added and incubated overnight. The primary antibodies used in this study were anti-mouse collagen type III (1:100; Calbiochem, San Diego, CA, USA) and anti-mouse collagen type I (1:100; Sigma-Aldrich, St. Louis, MO, USA). Then the membranes were incubated with biotinylated secondary anti-mouse and anti-rabbit antibodies followed by incubation with an avidin-biotin complex (ABC Elite $\mathrm{Kit}^{\mathrm{TM}^{\circledR}}$, Vector Laboratories, Burlingame, CA, USA) for $40 \mathrm{~min}$ at room temperature. Finally, the stained membranes were developed with the enzyme substrate 3,30-diaminobenzidine and hydrogen peroxide (DAB Substrate $\mathrm{Kit}^{\mathrm{TM}}{ }^{\circledR}$, Vector Laboratories, Burlingame, CA, USA) for $3 \mathrm{~min}$. The absorbance was measured at $450 \mathrm{~nm}$ using an ELISA reader (Infinite F200, TECAN, Crailsheim, Germany). The relative amount of collagen type I and type III deposited on the membranes was expressed as the percentage of the amount of corresponding protein on the conditioned membranes compared to that on the unconditional ones. For photograph, the indicated membranes were counter-stained with haematoxylin (Fisher Scientific, Pittsburgh, PA, USA) for $30 \mathrm{~s}$

\section{Results}

\subsection{Branching morphogenesis of SMG explants on PVA, chitosan and PC membranes}

To explore the branching pattern of SMG explants, SMG explants were cultured on PVA, chitosan, and PC membranes. In the culture system, the phenotypes and the developmental stages of SMG explants cultured on PC membranes were similar to those cultured on the floating PC filters as reported previously $[4,18]$ (data not shown). After $24 \mathrm{~h}$ in culture, the differences in SMG branching numbers were observed among PVA, chitosan, and PC (Fig. 1). The explants cultured on chitosan and PC started to branch but not on PVA. For another $24 \mathrm{~h}$, SMG explants on chitosan and PC proceeded successive branching, but the explants on PVA still remained unbranched. Furthermore, the budding number of explants on chitosan was significantly greater than that on PC after $48 \mathrm{~h}$ of culture $(p<0.01)$, although the morphologies of explants were similar.

\subsection{Extracellular matrix deposition on substrates after cultured with SMG explants}

To confirm whether the promoting effects of chitosan membranes originated from extracellular matrix deposition, the used membranes which had been cultured with explants were examined by SEM to demonstrate the morphological evidence of the presence of extracellular matrix deposition. On PVA membranes, neither extracellular matrix nor cells were left on the surface (Fig. 2(a)). Conversely, on chitosan membranes, some residual cells were adherent to the substrates. Between the cells, fibrillar matrices were observed intertwined on the surface (Fig. 2(b)). Nonetheless, on PC membranes, the extracellular matrix deposition was restricted within the surroundings of cells (Fig. 2(c)). The results indicated that when SMG explants were cultured on the chitosan membranes, much fibrillar extracellular matrices cohered to the surface. The extracellular matrices

\section{a}
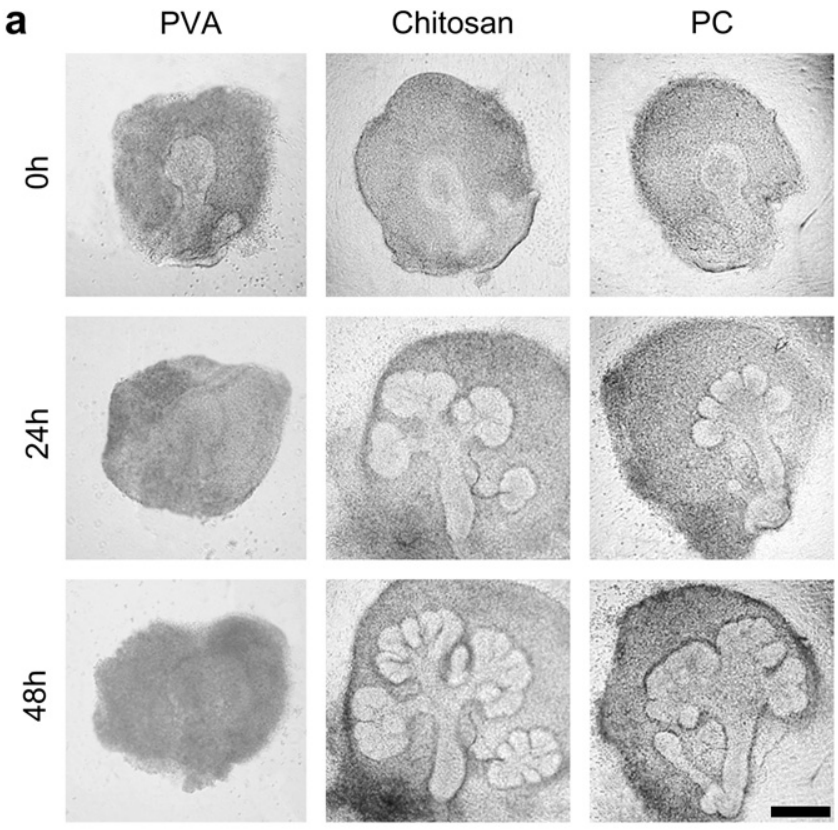

b

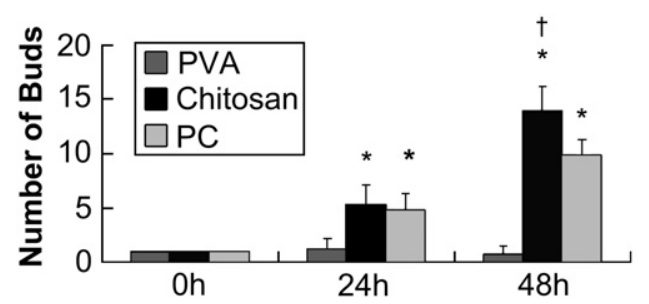

Fig. 1. (a) Branching morphogenesis of SMG on PVA, chitosan, and PC after 0,24 , and $48 \mathrm{~h}$ in culture. Scale bars $=100 \mu \mathrm{m}$. (b) The budding numbers measured at 0,24 and $48 \mathrm{~h}$. Asterisk $(*)$ denotes significant differences $(p<0.001)$ compared with PVA, while the cross $(\dagger)$ denotes significant differences compared with PC $(p<0.01$, paired $t$-test $)$.

distributed in a reticular manner and formed a mesh-like pattern. In addition, the fibrils extended further beyond the extent of cells (Fig. 2(d)), and numerous thin filaments seemed to coalesce into thick fibers (Fig. 2(e)). The results suggested that chitosan membrane provided a more favorable environment for SMG extracellular matrix deposition and organization.

\subsection{SMG branching on conditioned membranes}

Next, the conditioned membranes of PVA, chitosan, and PC, which had been used for SMG explants' culture, were prepared to further clarify the effects of used substrates on SMG branching. As shown in Fig. 3(a), the explants cultured on the conditioned PVA membranes were still undeveloped. The cultured SMG explants on conditioned PC membranes partly increased budding numbers as compared with those on the unconditioned membranes. Nonetheless, those on conditioned chitosan substrates showed more branches. In addition, the ratios of SMG buds between conditioned and unconditioned membranes were analyzed, which significantly increased on the chitosan membrane than those on the PC substrates 

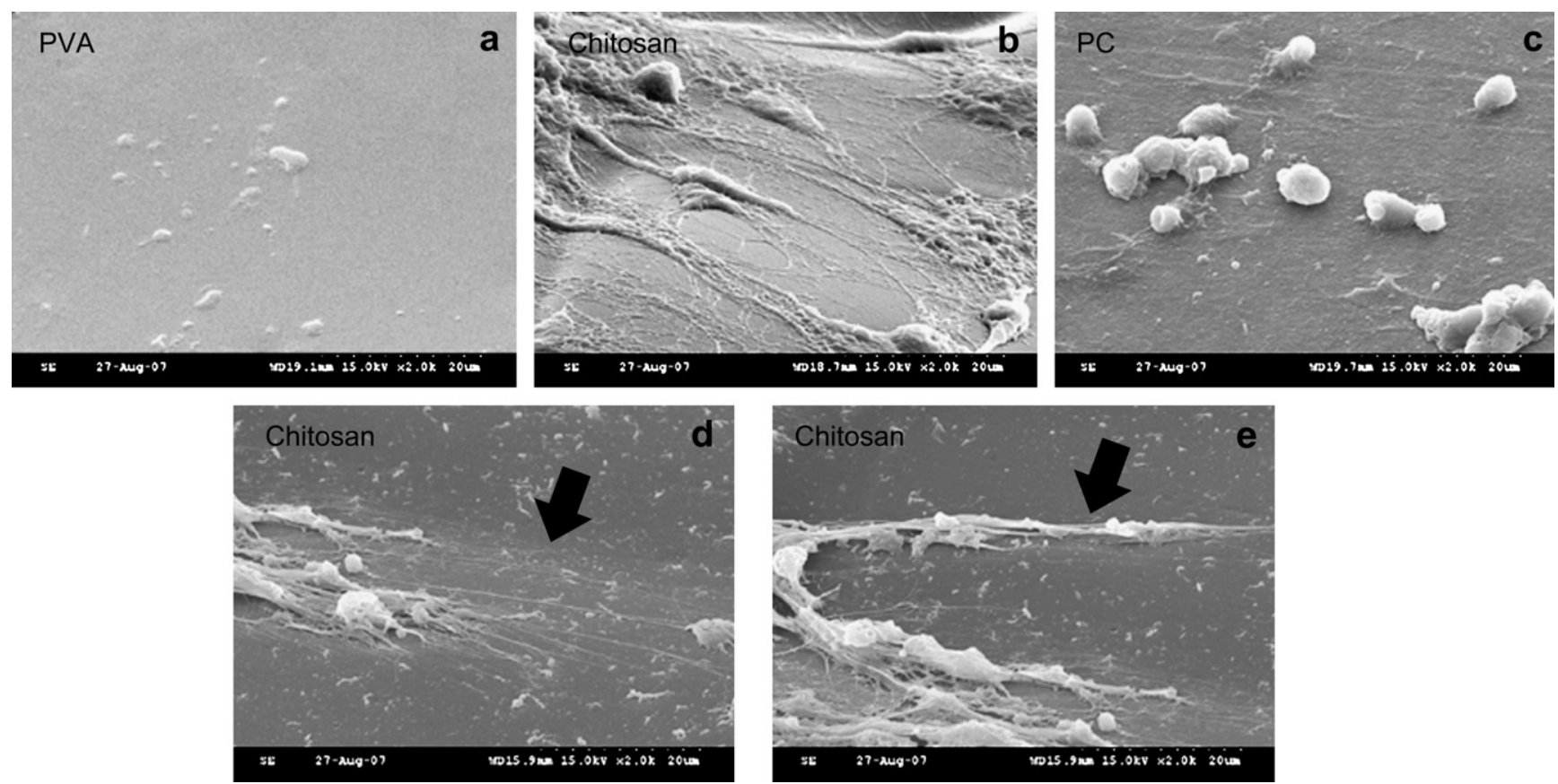

Fig. 2. Scanning electron micrographs of conditioned membranes of (a) PVA, (b) chitosan, and (c) PC. The micrographs of chitosan conditioned membranes showed the extracellular matrix extended in (d) a fibrillar pattern or (e) broad fibers (arrows: fibrils of deposited extracellular matrix).

(Fig. 3(b)). The results suggested that the conditioned chitosan membranes which had been cultured with SMG explants were able to exert a better promoting effect on the SMG branching.

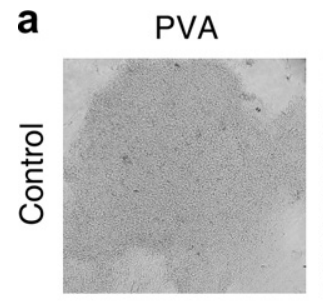

Chitosan
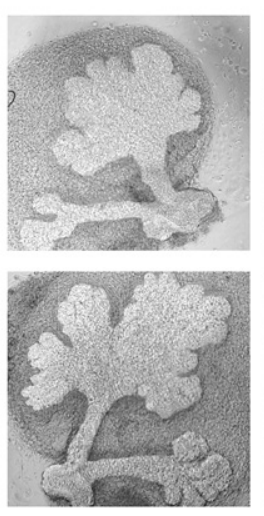

b

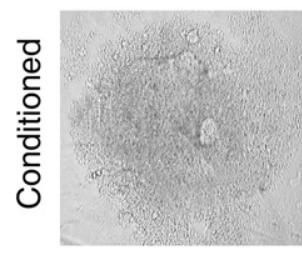

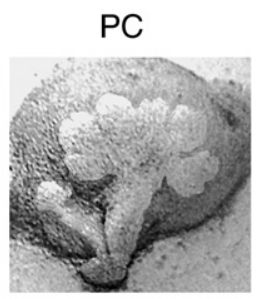

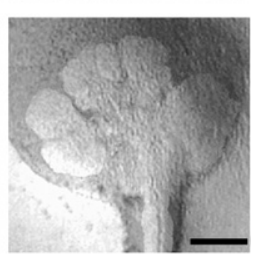

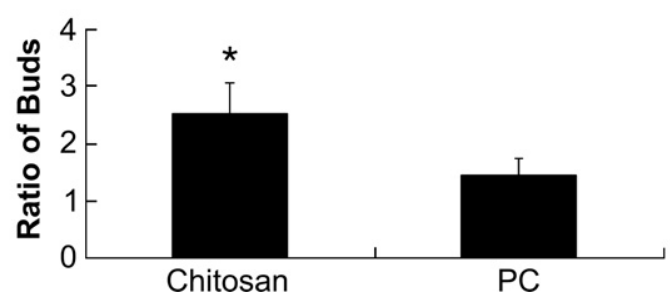

Fig. 3. (a) The effects of PVA, chitosan, and PC conditioned membranes on SMG branching morphogenesis (upper panels: SMG explants cultured on control membrane; lower panels: SMG explants cultured on conditioned membrane). Scale bars $=100 \mu \mathrm{m}$. (b) The ratio of buds presented as the budding numbers of SMGs on conditioned membrane compared to that on indicated control membranes after $48 \mathrm{~h}$ of culture. Asterisk $(*)$ denotes significant differences compared with PC $(p<0.001$, paired $t$-test $)$.

\subsection{SMG branching on conditioned membranes treated with collagenase}

To confirm whether the promoting effects of conditioned chitosan membranes originated from extracellular matrix deposition, especially collagen, the conditioned membranes were treated with collagenase before being applied for subsequent SMG culture. After being cultured on the collagenasetreated conditioned membranes, the SMG explants exhibited similar branching phenotypes as compared with those on unconditioned PC membranes, indicating that the transient collagenase treatment was not obviously pernicious to the ex vivo development of cultured SMG explants. Similarly, the explants on PVA remained unbranched as usual. On the collagenasetreated conditioned chitosan membranes, SMG explants demonstrated the same branching phenotypes as compared with those on the unconditioned chitosan membranes (Fig. 4(a)). In the analysis of SMG budding ratio between the conditioned membranes treated with collagenase and the unconditioned membranes, no differences were found in the buds' ratio between PC and chitosan (Fig. 4(b)). The results indicated that the branching-promoting effects of chitosan membrane were eliminated by collagenase, suggesting the factors presumably originated from the deposition of collagen on the conditioned chitosan membranes.

\subsection{Expression and quantification of collagen on conditioned membranes}

Since the branching-promoting effects of conditioned chitosan membranes were eliminated by collagenase, the immunohistochemical assays were performed to identify the presence of collagen, which was expressed in the SMG branching 
a
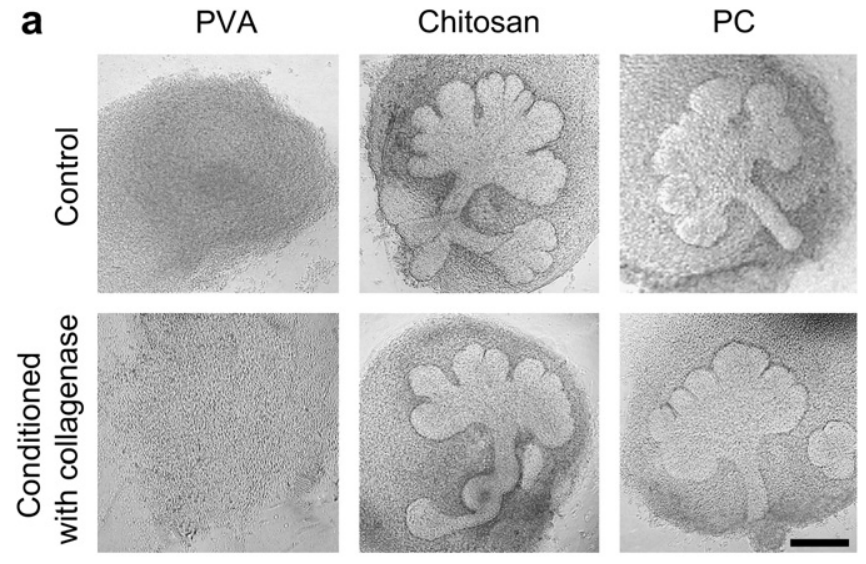

b

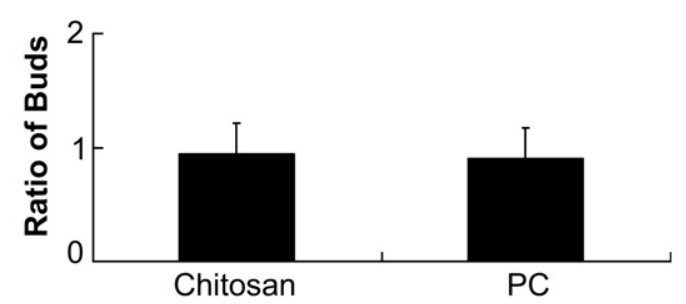

Fig. 4. (a) The effects of PVA, chitosan, and PC conditioned membranes treated with collagenase on SMG branching morphogenesis (upper panels: SMG explants cultured on control membrane; lower panels: SMG explants cultured on conditioned membrane treated with collagenase). Scale bars $=100 \mu \mathrm{m}$. (b) The ratio of buds presented as the budding numbers of SMG explants on the conditioned membrane treated with collagenase compared to that on the indicated control membranes after $48 \mathrm{~h}$ of culture. No significant difference was noted between SMG branching ratio on chitosan and PC ( $p>0.05$, paired $t$-test).

morphogenesis [19]. The deposited extracellular matrices on the conditioned membranes after $48 \mathrm{~h}$ culture were stained and the results are shown in Fig. 5. The unconditioned membranes of different substrates were first examined, and the results were regarded as background (data not shown). By type I collagen antibody staining, some reticular patterns were detected on the conditioned chitosan membranes (Fig. 5(a)), indicating that the presence of type I collagen within the deposited extracellular matrices. On the contrary, although some adherent residual cells were noted, scanty type I collagen was detected on the conditioned PC surface (Fig. 5(a)). Furthermore, on PVA, no matter whether the membranes conditioned or not, neither cells nor extracellular matrix was noted (Fig. 5(a)). Quantitatively, the deposition of type I collagen on the conditioned chitosan membranes significantly increased compared to that on PVA $(p<0.001$, paired $t$-test $)$ (Fig. 5(c)). The results provided evidence that type I collagen was present in the deposited extracellular matrices on the conditioned chitosan membranes.

Similarly, by using type III collagen antibody, some fibrillar patterns of staining were seen on the conditioned chitosan membranes (Fig. 5(b)). On conditioned PC membranes, some residual cells with scanty deposited extracellular matrices surrounding the cells were detected while none were observed on PVA conditioned membranes (Fig. 5(b)). In the quantitative analysis, only the amount of deposited type III collagen on the conditioned chitosan membranes increased significantly compared to others $(p<0.001$, paired $t$-test $)$ (Fig. 5(d)). Thus, the results indicated that type III collagen was also present within the deposited extracellular matrices on the conditioned chitosan membranes. These staining evidence suggested that the presence of type I and type III collagen was likely to contribute to the branching-promoting effects of chitosan membranes.

\section{Discussion}

It is well documented that the physical and chemical characteristics of material surface govern cellular responses and ultimately affect the success of tissue engineering applications. In this work, chitosan membranes were used for the first time to study their impact on SMG branching. Compared to PVA and PC, it was found that chitosan provided a more preferential environment for salivary gland branching morphogenesis. Previously, the criteria to select substrata for investigating branching morphogenesis principally prefer those with inert biological properties, which provide only structural support without an interference of endogenous cellular behaviors. Therefore, PC has been widely used as a culture material for decades to investigate SMG branching morphogenesis. Nonetheless, the concept of scaffolds biocompatibility has evolved greatly, from a material with inert biological properties to a bioactive matrix which serves as a dynamic environment for tissue development. Therefore, some natural and synthetic materials have been proposed and examined for their possible applications in the regenerative branching morphogenesis $[18,20,21]$. Nonetheless, their clinical applications might be limited because of the biocompatible issues. On the other hand, chitosan, which is free from these drawbacks and easily tailored for clinical demand, might be the potential substratum. Chitosan not only provides a preferential growth environment for salivary cells [15], but also presents as a promising carrier for angiogenetic factors to control vascularization $[22,23]$. During the salivary organogenesis, the development of ramified vasculature is in accordance with the establishment of epithelial branches [2]. Therefore, by applying chitosan substratum, the progress toward the regeneration of a functional salivary tissue might be accelerated.

It has been reported that the substrata conditioned by precultured cells can absorb required factors for subsequent cultured cells' adhesion and spreading [24]. By staining methods, these factors are presented in a fibrillar pattern beneath the cells as footprints on the substratum [24]. It is well known that cell-substrate adhesion is a multi-step process which initiates by absorption of particular components of extracellular matrix, such as collagen, fibronectin, and laminin, onto the substrate surface. Thus, biomaterial surface characteristics might affect cellular response through the absorptive protein layer, by increasing the amount of protein absorption and the arrangement of deposited configuration. In this study, the serum-free medium was used, so the branching-promoting effects of chitosan membranes were attributed to the extracellular matrix secreted from SMG explants, which was induced 
a
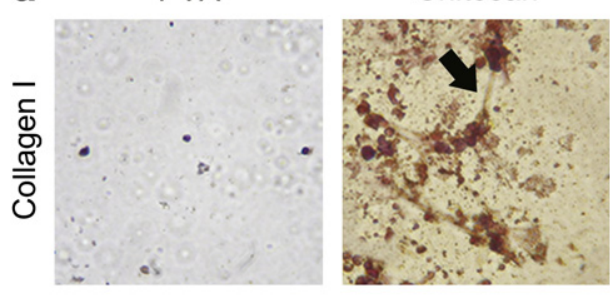

b

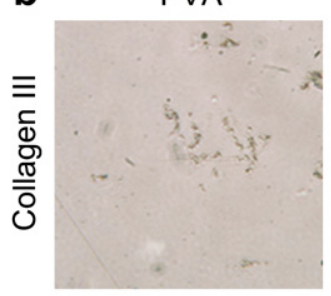

Chitosan

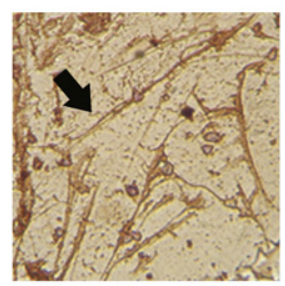

PC

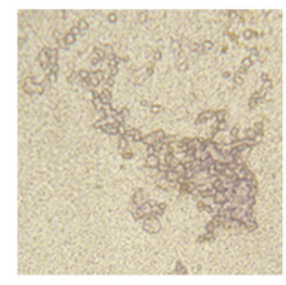

PC

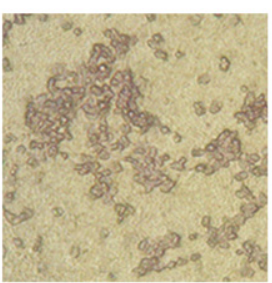

C

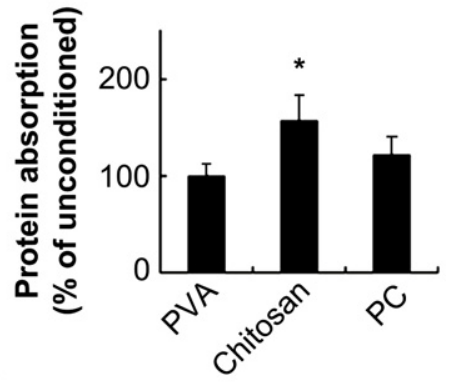

d

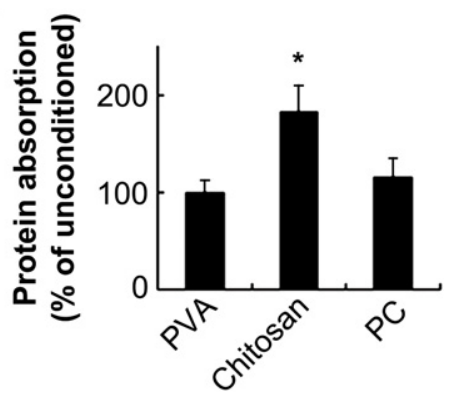

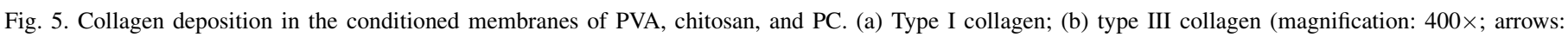

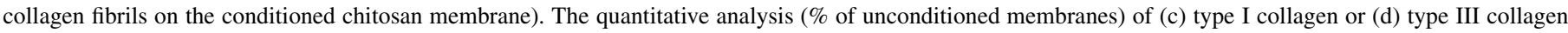
deposition on the conditioned membranes. Asterisk $(*)$ denotes a significant difference compared with PVA ( $p<0.001$, paired $t$-test).

by the direct contact with prepared substrates. In general, the ideal scaffolds for tissue regeneration are to provide a favorable environment for extracellular matrix deposition. In the current study, abundant fibrillar extracellular matrix can be observed intertwined between adherent salivary gland cells on conditioned chitosan membranes, which were not observed in PC and PVA substrata (Fig. 2). Therefore, chitosan is presumably to be a more bioactive material for salivary glands' branching.

Because of the abolishing effect of collagenase and the presence of type I and type III collagen (Figs. 4 and 5), it is reasonable to assume that collagen plays an important role in the conditioned chitosan membrane to facilitate SMG branching. During salivary gland development, collagen is an important extracellular matrix for salivary gland branching [25]. Firstly, type I collagen is widely distributed over the whole mesenchyma and the epithelial-mesenchymal interfaces [19], though with less branching-promoting effects [26]. Similarly, in the mesenchyma, type III collagen is expressed in the same fashion as that of type I collagen, but accumulates more intensively at every cleft bottom [19]. The specific expression pattern is essential for salivary gland branching morphogenesis [25,27]. There are several hypotheses accounting for the underlying mechanisms for collagen in promoting SMG branching. It has been proposed that the clefts and branch formation stem from the collagen fibers' contraction and alignment [28]. The mesenchymal contraction of collagen fibrils at the cleft point where epithelium is associated with more plastic properties accounts for the mechanism that drives the deepening of cleft and forms epithelial branches [29]. Furthermore, it is suggested that the deposition of collagen fiber in a newly formed cleft may be necessary for the stabilization of initial branch formation [27]. Accordingly, in the successive SMG branching, the existence of collagen is requisite.

One possibility accounting for the deposition of abundant collagen fibers on conditioned chitosan membrane is that chitosan is capable of stimulating SMG explants to excrete more collagen fibers. Chitosan has been demonstrated to have stimulatory effects on extracellular matrix production of cells, and is capable of inducing collagen fibers' formation [30,31]. Type I, type III, and type IV collagen synthesis are enhanced in the presence of chitosan-related substances [32,33]. Accordingly, the accelerating effects of chitosan on wound healing are proved to result from collagen synthesis, especially type III collagen [33]. Similarly, chondrocytes cultured on chitosan substrata are capable of synthesizing cartilage-specific collagens, which consequently enhance chondrogenesis [34]. Together, chitosan provides an appropriate environment for facilitating cells to secret essential extracellular matrix for tissue-specific requirement. In salivary gland, these effects might be beneficial to branching morphogenesis of SMG.

In addition to the stimulating effects of chitosan on SMG collagen production, the second possibility is that chitosan has a better ability to absorb collagen. Many applications of chitosan stem from its structural characteristics, which might be helpful in developing complexes with many extracellular matrices, such as glycosaminoglycans and collagens [35,36]. Clinically, chitosan has been well known for its positive influence on wound healing because of the increasing collagen deposition [33,37]. By covering wounds with chitosan, the newly formed collagen fibers can be observed and are oriented in an organized manner [38]. Accordingly, by culturing SMG on chitosan membranes, it is possible that the secreted collagen is easily deposited and aligned in a tissue-specific manner, which might be helpful to the branch formation of SMG. 
Certainly, it is evident that the accumulation of collagen in the interface between epithelium and mesenchyma might be more directly beneficial to the formation of SMG branches [19]. Nonetheless, in the current culture system, the possibility that the epithelium is in direct contact with the substrata could not be completely excluded [16]. Moreover, mesenchymal cells have been proved to be able to produce more organized collagen when they grow in the presence of collagen $[39,40]$. Therefore, whether the branching-promoting effect of deposited collagen directly influences the epithelium or indirectly affects the mesenchymal cells needs further investigation.

Based on this study, although SMG has been routinely cultured on PC for decades, it sprouts fewer branches than that of in vivo development by the same stage [2]. On the conditioned PC membrane assay, many cells were adherent to the membranes. However, compared to chitosan membranes, lesser extracellular matrix deposition and scanty collagen synthesis could be detected on the surface, suggesting that PC was not as bioactive as chitosan membranes. Similarly, PVA, a relatively hydrophilic substratum, is unable to absorb abundant extracellular matrix and fails in keeping cell attachment and survival, finally hampering the branching processes. A wet environment is straightforwardly regarded as more favorable for salivary cells because of their roles in saliva secretion in vivo. Nonetheless, an extremely hydrophilic environment is not thought to be an appropriate substratum for promoting SMG branching.

\section{Conclusion}

The cultured scaffold composed of chitosan substrates demonstrates a preferential characteristic of enhancing salivary gland branching. By interacting with SMG explants, chitosan is competent to induce cells to synthesize and deposit the collagen-related extracellular matrix on the chitosan surface, which is essential for subsequent branch formation. Therefore, for the first time, chitosan has been proven to be an appropriate biomaterial to promote SMG branching. The current study provides evidence that chitosan substratum is bioactive in SMG branching morphogenesis and suggests that chitosan might be capable of recapitulating salivary gland branching, which is useful for the scaffold design of regenerative salivary glands.

\section{Acknowledgements}

The authors thank National Science Council of the Republic of China and National Taiwan University Hospital for their financial support of this research.

\section{References}

[1] Metzger RJ, Krasnow MA. Genetic control of branching morphogenesis. Science 1999;284:1635-9.

[2] Patel VN, Rebustini IT, Hoffman MP. Salivary gland branching morphogenesis. Differentiation 2006;74:349-64.
[3] Jaskoll T, Chen H, Min Zhou Y, Wu D, Melnick M. Developmental expression of survivin during embryonic submandibular salivary gland development. BMC Dev Biol 2001;1:5.

[4] Hoffman MP, Kidder BL, Steinberg ZL, Lakhani S, Ho S, Kleinman HK, et al. Gene expression profiles of mouse submandibular gland development: FGFR1 regulates branching morphogenesis in vitro through BMP- and FGF-dependent mechanisms. Development 2002;129:5767-78.

[5] Joraku A, Sullivan CA, Yoo JJ, Atala A. Tissue engineering of functional salivary gland tissue. Laryngoscope 2005;115:244-8.

[6] Vissink A, Jansma J, Spijkervet FK, Burlage FR, Coppes RP. Oral sequelae of head and neck radiotherapy. Crit Rev Oral Biol Med 2003;14:199-212.

[7] Warde P, O'Sullivan B, Aslanidis J, Kroll B, Lockwood G, Waldron J, et al. A Phase III placebo-controlled trial of oral pilocarpine in patients undergoing radiotherapy for head-and-neck cancer. Int J Radiat Oncol Biol Phys 2002;54:9-13.

[8] Chen MH, Hsu YH, Lin CP, Chen YJ, Young TH. Interactions of acinar cells on biomaterials with various surface properties. J Biomed Mater Res A 2005;74:254-62.

[9] Aframian DJ, Cukierman E, Nikolovski J, Mooney DJ, Yamada KM, Baum BJ. The growth and morphological behavior of salivary epithelial cells on matrix protein-coated biodegradable substrata. Tissue Eng 2000;6:209-16.

[10] Khor E, Lim LY. Implantable applications of chitin and chitosan. Biomaterials 2003;24:2339-49.

[11] Suh JK, Matthew HW. Application of chitosan-based polysaccharide biomaterials in cartilage tissue engineering: a review. Biomaterials 2000;21:2589-98.

[12] Aspden TJ, Mason JD, Jones NS, Lowe J, Skaugrud O, Illum L. Chitosan as a nasal delivery system: the effect of chitosan solutions on in vitro and in vivo mucociliary transport rates in human turbinates and volunteers. J Pharm Sci 1997;86:509-13.

[13] Shi C, Zhu Y, Ran X, Wang M, Su Y, Cheng T. Therapeutic potential of chitosan and its derivatives in regenerative medicine. J Surg Res 2006;133:185-92.

[14] Di Martino A, Sittinger M, Risbud MV. Chitosan: a versatile biopolymer for orthopaedic tissue-engineering. Biomaterials 2005;26:5983-90.

[15] Chen MH, Chen RS, Hsu YH, Chen YJ, Young TH. Proliferation and phenotypic preservation of rat parotid acinar cells. Tissue Eng 2005;11:526-34.

[16] Takahashi Y, Nogawa H. Branching morphogenesis of mouse salivary epithelium in basement membrane-like substratum separated from mesenchyme by the membrane filter. Development 1991;111:327-35.

[17] Gong HP, Zhong YH, Li JC, Gong YD, Zhao NM, Zhang XF. Studies on nerve cell affinity of chitosan derived materials. J Biomed Mater Res 2000;52:285-95.

[18] Wei C, Larsen M, Hoffman MP, Yamada KM. Self-organization and branching morphogenesis of primary salivary epithelial cells. Tissue Eng 2007;13:721-35.

[19] Nakanishi Y, Nogawa H, Hashimoto Y, Kishi J, Hayakawa T. Accumulation of collagen III at the cleft points of developing mouse submandibular epithelium. Development 1988;104:51-9.

[20] Mondrinos MJ, Koutzaki S, Jiwanmall E, Li M, Dechadarevian JP, Lelkes PI, et al. Engineering three-dimensional pulmonary tissue constructs. Tissue Eng 2006;12:717-28.

[21] Joraku A, Sullivan CA, Yoo J, Atala A. In-vitro reconstitution of threedimensional human salivary gland tissue structures. Differentiation 2007;75:318-24.

[22] Fujita M, Ishihara M, Simizu M, Obara K, Ishizuka T, Saito Y, et al. Vascularization in vivo caused by the controlled release of fibroblast growth factor-2 from an injectable chitosan/non-anticoagulant heparin hydrogel. Biomaterials 2004;25:699-706.

[23] Ishihara M, Obara K, Nakamura S, Fujita M, Masuoka K, Kanatani Y, et al. Chitosan hydrogel as a drug delivery carrier to control angiogenesis. J Artif Organs 2006;9:8-16.

[24] Grinnell F, Feld MK. Initial adhesion of human fibroblasts in serum-free medium: possible role of secreted fibronectin. Cell 1979;17:117-29. 
[25] Nakanishi Y, Uematsu J, Takamatsu H, Fukuda T, Yoshida K. Removal of heparan sulfate chains halted epithelial branching morphogenesis of the developing mouse submandibular gland in vitro. Dev Growth Differ 1993;35:371-84.

[26] Kratochwil K, Dziadek M, Lohler J, Harbers K, Jaenisch R. Normal epithelial branching morphogenesis in the absence of collagen I. Dev Biol 1986;117:596-606.

[27] Grobstein C, Cohen J. Collagenase: effect on the morphogenesis of embryonic salivary epithelium in vitro. Science 1965;150:626-8.

[28] Nakanishi Y, Sugiura F, Kishi J, Hayakawa T. Scanning electron microscopic observation of mouse embryonic submandibular glands during initial branching: preferential localization of fibrillar structures at the mesenchymal ridges participating in cleft formation. J Embryol Exp Morphol 1986;96:65-77.

[29] Umeda Y, Miyazaki Y, Shiinoki H, Higashiyama S, Nakanishi Y, Hieda Y. Involvement of heparin-binding EGF-like growth factor and its processing by metalloproteinases in early epithelial morphogenesis of the submandibular gland. Dev Biol 2001;237:202-11.

[30] Okamoto Y, Shibazaki K, Minami S, Matsuhashi A, Tanioka S, Shigemasa Y. Evaluation of chitin and chitosan on open would healing in dogs. J Vet Med Sci 1995;57:851-4.

[31] Kojima K, Okamoto Y, Kojima K, Miyatake K, Fujise H, Shigemasa Y, et al. Effects of chitin and chitosan on collagen synthesis in wound healing. J Vet Med Sci 2004;66:1595-8.

[32] Kojima K, Okamoto Y, Miyatake K, Kitamura Y, Minami S. Collagen typing of granulation tissue induced by chitin and chitosan. Carbohydr Polym 1998;37:109-13.
[33] Ueno H, Yamada H, Tanaka I, Kaba N, Matsuura M, Okumura M, et al. Accelerating effects of chitosan for healing at early phase of experimental open wound in dogs. Biomaterials 1999;20:1407-14.

[34] Sechriest VF, Miao YJ, Niyibizi C, Westerhausen-Larson A, Matthew HW, Evans $\mathrm{CH}$, et al. GAG-augmented polysaccharide hydrogel: a novel biocompatible and biodegradable material to support chondrogenes. J Biomed Mater Res 2000;49:534-41.

[35] Denuziere A, Ferrier D, Damour O, Domard A. Chitosan-chondroitin sulfate and chitosan-hyaluronate polyelectrolyte complexes: biological properties. Biomaterials 1998;19:1275-85.

[36] Taravel MN, Domard A. Collagen and its interactions with chitosan: III. Some biological and mechanical properties. Biomaterials 1996; 17:451-5.

[37] Hamilton V, Yuan Y, Rigney DA, Puckett AD, Ong JL, Yang Y, et al. Characterization of chitosan films and effects on fibroblast cell attachment and proliferation. J Mater Sci Mater Med 2006; 17:1373-81

[38] Mi FL, Shyu SS, Wu YB, Lee ST, Shyong JY, Huang RN. Fabrication and characterization of a sponge-like asymmetric chitosan membrane as a wound dressing. Biomaterials 2001;22:165-73.

[39] Bard JB, Higginson K. Fibroblast-collagen interactions in the formation of the secondary stroma of the chick cornea. J Cell Biol 1977;74:816-27.

[40] Doane KJ, Babiarz JP, Fitch JM, Linsenmayer TF, Birk DE. Collagen fibril assembly by corneal fibroblasts in three-dimensional collagen gel cultures: small-diameter heterotypic fibrils are deposited in the absence of keratan sulfate proteoglycan. Exp Cell Res 1992;202:113-24. 